\title{
Flight Hydrogen Sensor for use in the ISS Oxygen Generation Assembly
}

\author{
George Msadoques \\ Hamilton Sundstrand Space Systems International \\ Dr. Darby Makel \\ Makel Engineering, Inc.
}

\begin{abstract}
This paper provides a description of the hydrogen sensor Orbital Replacement Unit (ORU) used on the Oxygen Generation Assembly (OGA), to be operated on the International Space Station (ISS). The hydrogen sensor ORU is being provided by Makel Engineering, Inc. (MEI) to monitor the oxygen outlet for the presence of hydrogen. The hydrogen sensor ORU is a triple redundant design where each sensor converts raw measurements to actual hydrogen partial pressure that is reported to the OGA system controller. The signal outputs are utilized for system shutdown in the event that the hydrogen concentration in the oxygen outlet line exceeds the specified shutdown limit. Improvements have been made to the Micro-ElectroMechanical Systems (MEMS) based sensing element, screening, and calibration process to meet OGA operating requirements. Two flight hydrogen sensor ORUs have successfully completed the acceptance test phase. This paper also describes the sensor's performance during acceptance testing, additional tests planned to extend the operational performance calibration cycle, and integration with the OGA system.
\end{abstract}

\section{INTRODUCTION}

Hamilton Sundstrand Space Systems International, Inc. (HSSSI) is under contract to
NASA Marshall Space Flight Center (MSFC) to develop an Oxygen Generation Assembly (OGA). The OGA electrolyzes potable water from the Water Recovery System (WRS) to provide gaseous oxygen to the Space Station module atmosphere for metabolic consumption by crew and biological specimens. As a byproduct, gaseous hydrogen is generated. Under normal operating conditions, the hydrogen is vented to space or recovered by a Sabatier system which combines the hydrogen with carbon dioxide removed from the cabin air, providing a supplemental water source for the ISS. Under certain failure conditions of the OGA electrolysis cell stack, hydrogen could enter the oxygen outlet stream and be delivered to the cabin, creating a hazard. A Hydrogen Sensor Orbital Replacement Unit (ORU) consisting of three separate hydrogen sensors has been incorporated in the OGA design in the oxygen outlet line (Figure 1). It functions as a safety device to sense the presence of hydrogen, causing the controller to shut down the system. The three sensors operate independently, providing triple redundancy for system shutdown

The hydrogen sensor is a thin-film design based on metal-oxide-semiconductor sensing devices. It utilizes a palladium chrome $(\mathrm{Pd} / \mathrm{Cr})$ alloy as a catalyst to disassociate molecular hydrogen. The primary element of the design is a $\mathrm{Pd} / \mathrm{Cr}$ diode which has electrical properties that change when exposed to atomic hydrogen. The 
diode responds to low hydrogen concentrations and can accurately sense hydrogen partial pressures ranging from 0 to 2026 pascal (about 0 to $2 \%$ ).

\section{FLIGHT HARDWARE DESCRIPTION}

The flight hydrogen sensor ORU consists of three independent hydrogen sensors that are housed in-line in a stainless steel manifold containing a flow chamber for the oxygen stream being monitored. Figure 2 shows the sensor system containing three sensors in a manifold and an enclosure that contains the electronics to provide power and process the signal. The overall dimensions of the manifold and enclosure are 8.4 " wide $\times 4.00$ " deep $\times 5.25$ " tall (ORU is $8.4 " \times 4.15^{\prime \prime} \times 6.23$ " including the electrical receptacle and quick disconnects). The mass is approximately 10.3 pounds. The ORU has been designed consistent with applicable International Space Station standards.

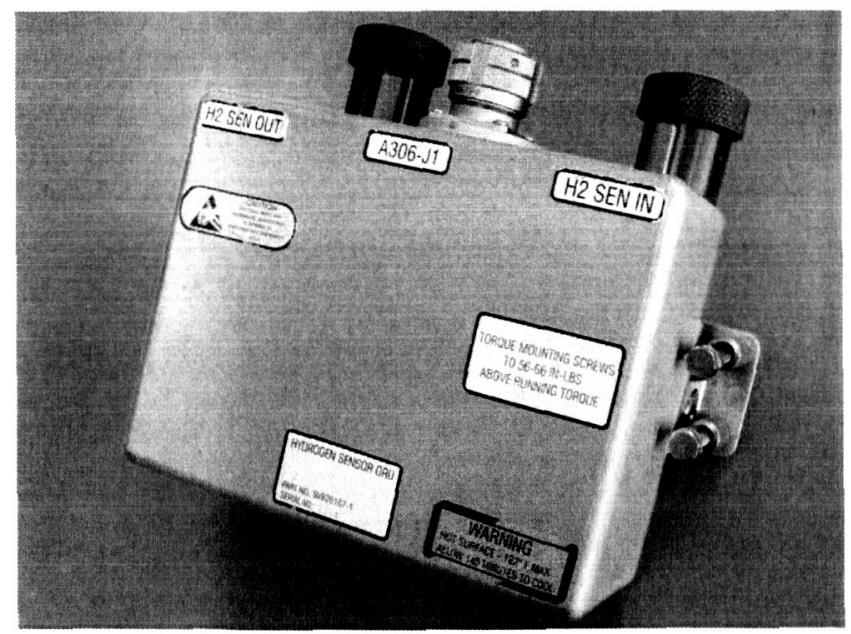

Figure 1. Flight Hydrogen Sensor Orbital Replaceable Unit.

The ORU is designed to allow replacement on orbit. The unit is mounted by four captive bolts that mount to the OGA Oxygen Outlet ORU manifold. The oxygen flow path inlet and outlets are attached by means of two quick disconnects.
The electronics for each independent sensor are on their own conduction cooled printed circuit board. Electronic components have been chosen to meet the reliability and radiation tolerance requirements of the program.
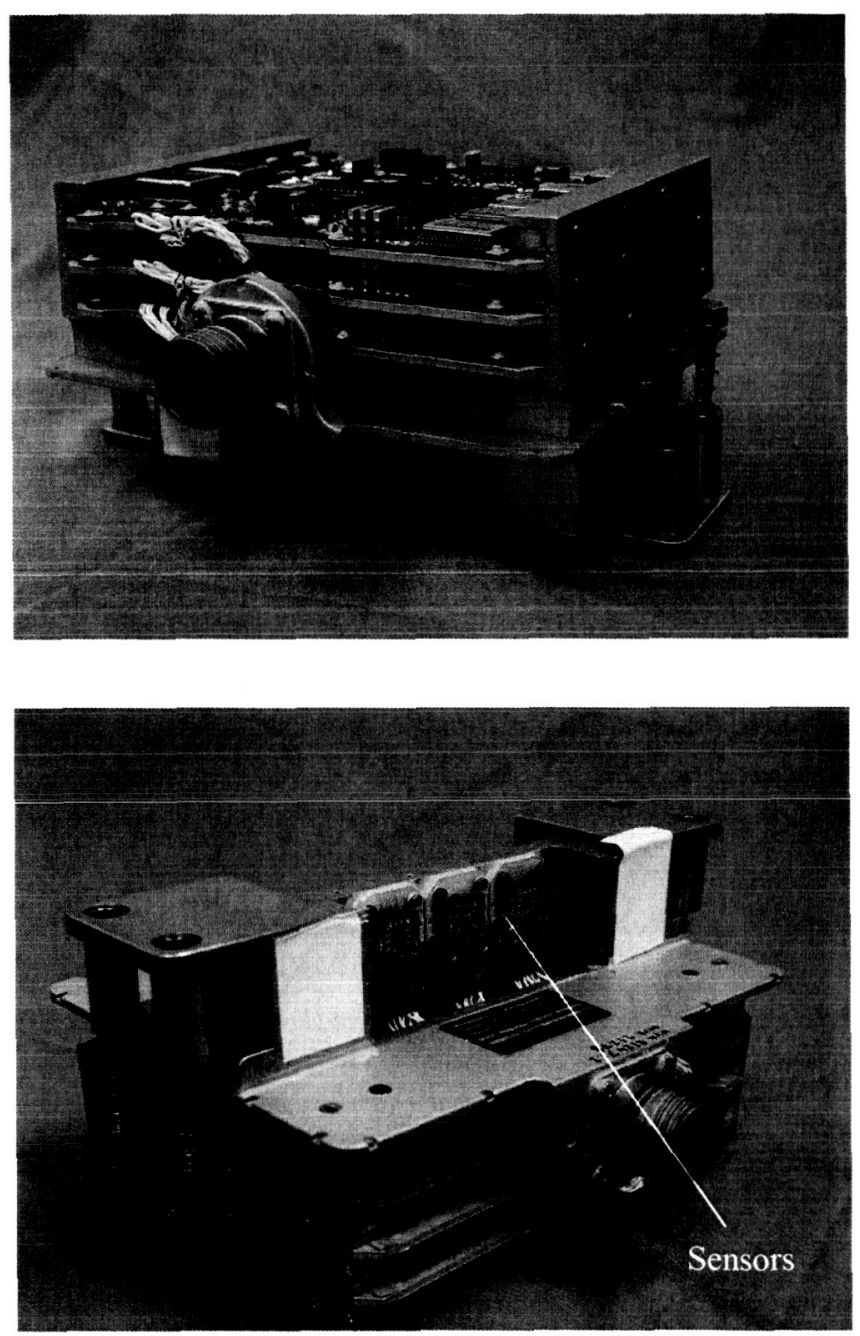

Figure 2. Flight Hydrogen Sensor ORU with Cover off showing Boards and Sensors

\section{TECHNOLOGY ADVANCEMENTS}

\section{SENSING TECHNOLOGY IMPROVEMENTS}

The MEMS based sensing technology used in the ORU was originally developed for detection of a leak in inert environments, therefore the OGA application required an extensive 
evaluation of the performance of the sensor under conditions representative of on-orbit OGA environment spanning a ten year system life. During the operating lifetime of the OGA system a wide range of gas temperature, dew point, background gas composition on system startup are expected to be encountered.

As the OGA system operating characteristics have become better understood, three key performance issues of the hydrogen sensor have been addressed. First is the operation in high humidity environment. Second is time response of the sensors and third is immunity to condensation. In order to ensure that sensors used for the ORU could achieve the desired operating characteristics, extensive screening of die is conducted at the subassembly level, a five point temperature calibration curve is used to accommodate for non-linearity and sensor uniqueness. Procedural changes were refined to optimize calibration.

Previous work on the system included an evaluation of performance over 180 days of continual operation in a dry oxygen background [3]. Following that testing, it was determined that dew point of the process oxygen stream from the OGA impacts the reading of the sensor. Additional system operating requirements that impact sensor accuracy were identified as variable background gas composition during normal, but infrequent flushing of the OGA system with nitrogen gas. Failure modes within the OGA can also result in an exposure to nitrogen. The Hydrogen Sensor ORU will accommodate this environment with a quick response during a hydrogen leak event.

Testing of the sensor has confirmed that the primary impact of water vapor in the process stream is related to the absolute. Over the ten year life of the OGA system the process stream dew point may vary from 60 to $85 \mathrm{~F}$ due to aging of the electrolysis cell stack. In order to reduce the sensitivity to humidity, two primary changes to the technology described in the Reference 3 paper were undertaken. First, use of overlapping sensing elements was dropped (i.e. a resistive sensing element was eliminated from the design) and the operating temperature of the diode sensing element was increased from $65 \mathrm{C}$ to approximately $80 \mathrm{C}$ resulting in reduced dew point variation as shown in Figure 3 . At the higher die operating temperature the resistive sensing element did not provide sufficient sensitivity for concentrations under $1 \%$ to justify its continued use.

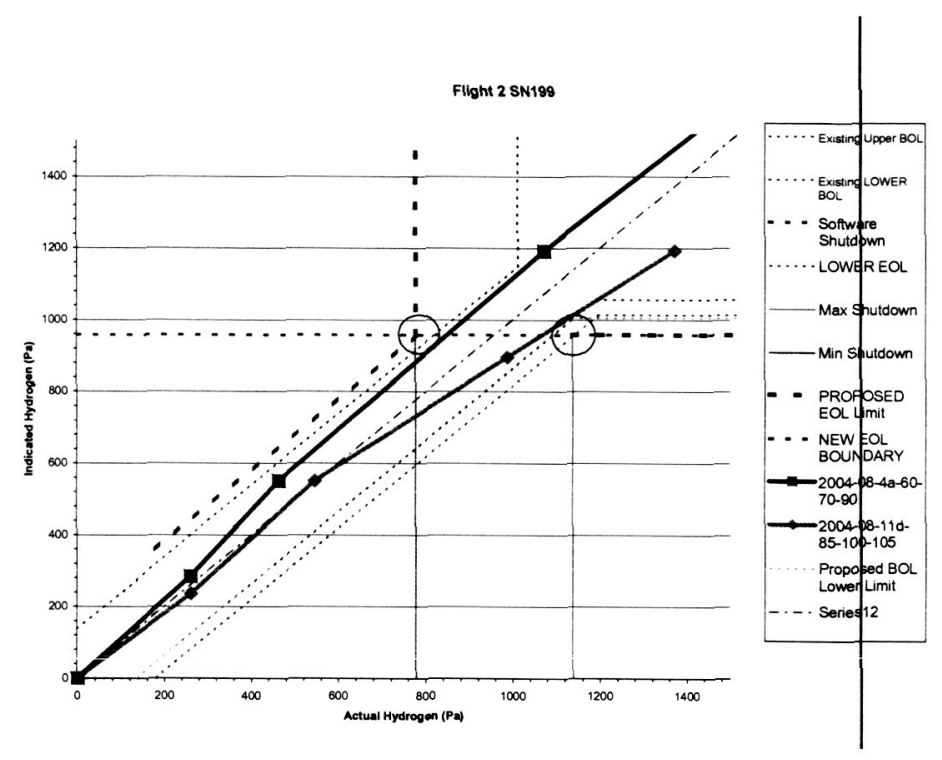

Figure 3. Effect of dew point on hydrogen signal in humidity hydrogen/oxygen process stream.

A key performance parameter for the sensor is time response to hydrogen leaks from the cell stack. To ensure that a flammable mixture cannot reach the crew cabin, the sensors are required to indicate a shutdown level of hydrogen (approximately 1\%) when exposed to a mixture at the lower explosive limit (LEL) of hydrogen (approximately 4\% Hydrogen) within six seconds. This time response ensures that the OGA system can close all required valves before any potentially hazardous mixtures are released into the crew cabin. Refer to the simplified schematic of the OGA schematic in Figure 4. Figure 5 shows a typical time response of the sensor to a step change in hydrogen at typical OGA operating conditions. In order to ensure an adequate yield from screened sensors the system requirement was adjusted from five seconds to six seconds. This relaxed requirement on the sensor did not affect overall system safety. 


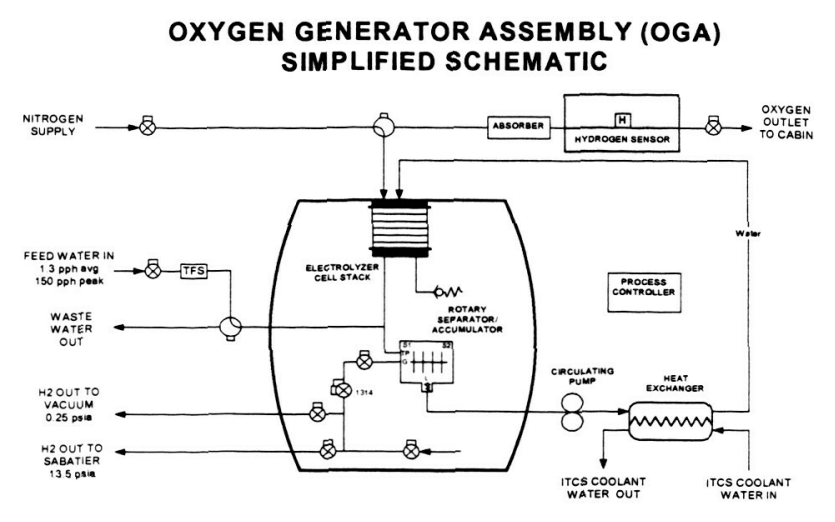

Figure 4 OGA Schematic with Hydrogen Sensor Shown

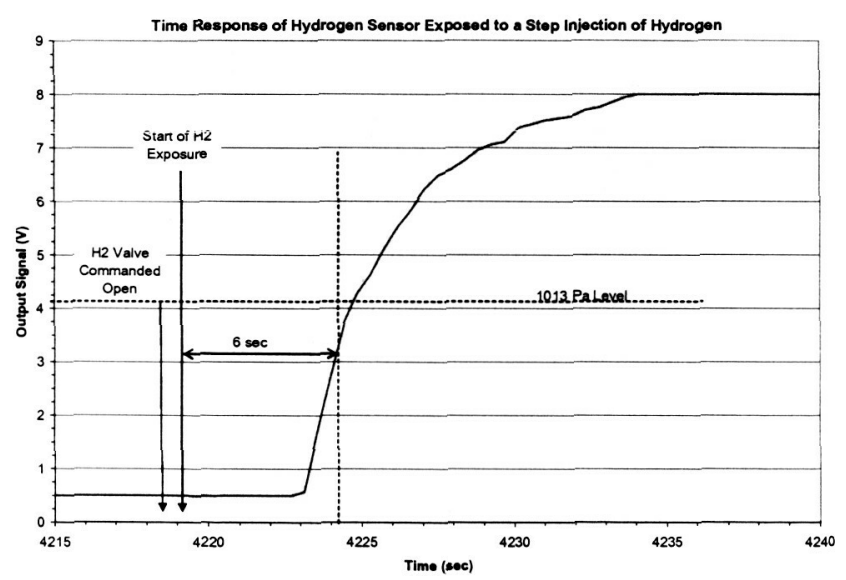

Figure 5. Typical Time Response of Hydrogen Sensor Exposed To A Step Change of Gas From $0 \%$ to $4 \%$ Hydrogen.

While condensation of water in the hydrogen sensor ORU is not possible under normal operating conditions, it has been determined that there exists the possibility for condensed water to exist in the ORU under certain OGA hardware shut down modes. Tests conducted to assess the impact of condensation on the sensor head showed the potential for damage from electrolysis on the energized elements on the sensor die. Figure 6 shows the results of bench testing to assess the interaction of liquid water on the surface of the sensing element.

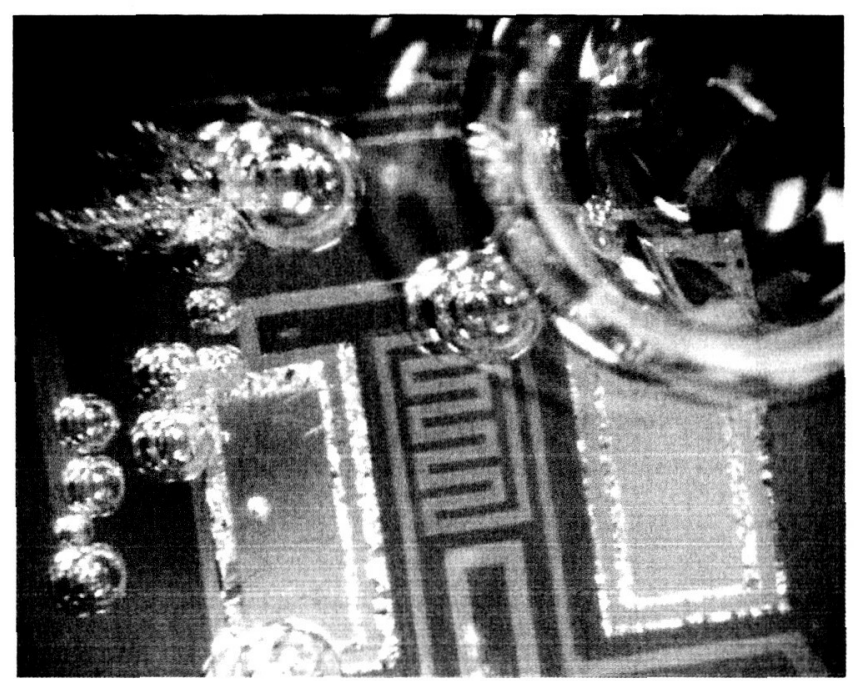

Figure 6. Bubble formation from electrolysis from the surface of the hydrogen sensor die.

Gas bubbles evolving from the surface indicate electrolysis from the electrodes operating at potentials up to 5 VDC which exist on the wetted surface of the die.

\section{FLIGHT AND QUAL UNIT STATUS}

Two flight ORUs had successfully completed acceptance testing at Makel Engineering in November 2004. The Qualification unit is in process and is expected to be completed in the fall of 2005. A significant part of the qualification test time relates to two 90 -day calibration cycles that demonstrate the ORU's ability to remain within its accuracy requirements.

Acceptance testing of the flight ORU includes random vibration testing, thermal cycle testing, electrical testing, accuracy and time response testing. Figure 7 shows one of the completed flight ORUs installed on a vibration test fixture during acceptance testing. 


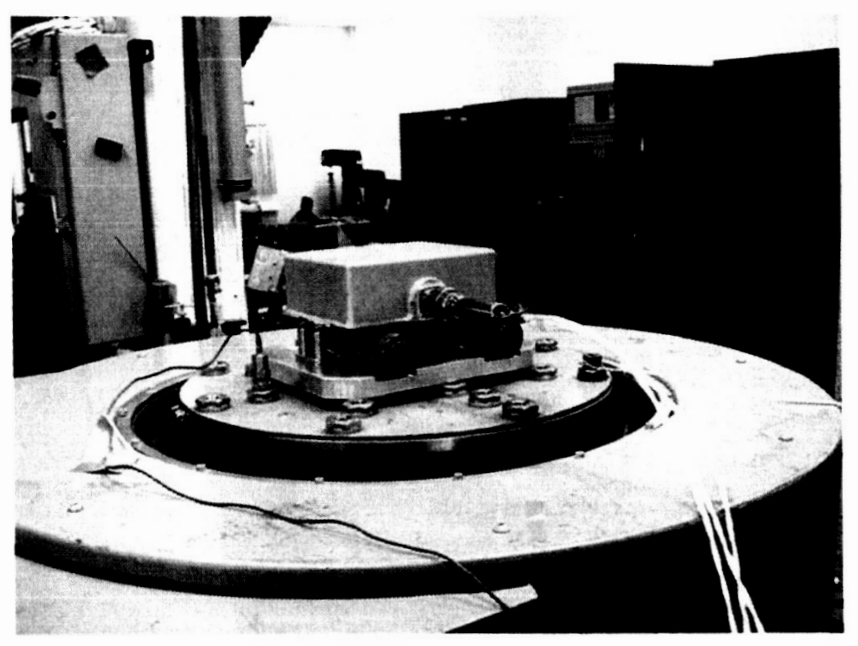

Figure 7. Flight ORU under going random vibration testing.

\section{RADIATON HARDNESS TESTING}

Electronic components have been selected such that they will function within specification during and after exposure to the expected ionization radiation environment. This selection was based on review of radiation test data on same of similar part to sufficiently predict part behavior in the radiation of the Space Station. In the case of the metal-oxide-semiconductor sensing device, the internal thin oxide layer can be susceptible to Single Event Gate Rupture (SEGR). Hence, the following tests were performed and the device successfully passed ion radiation testing.

Gamma Total lonizing Dose (TID) irradiation was tested at the Boeing Radiation Effects Laboratory (BREL) in Seattle, WA. The radiation source was a J.L. Shepherd 81-22/484 Irradiator (Co60 gamma irradiator), which was used to expose sensors to increasing levels of gamma radiation (Figure 8). Sensors were maintained at constant temperature using closed loop temperature control to simulate normal sensor operation. A lithium fluoride (LiF) dosimeter was used to calibrate the dose levels within the test fixture. Sensors were exposed to $1 \%$ hydrogen in air, then purged with air, prior to each radiation threshold. The radiation dose was provided at $10 \mathrm{rad}(\mathrm{Si}) / \mathrm{s}$, up to testing thresholds of .93 Krad, $1.85 \mathrm{Krad}, 3 \mathrm{Krad}$, and 5 Krad. In all cases, sensors showed no significant change in performance.

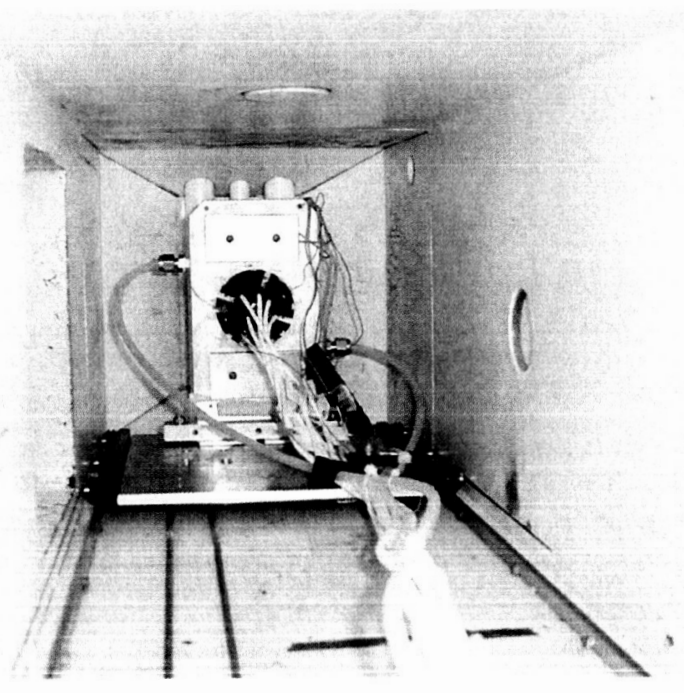

Figure 8: J.L. Shepherd $\mathrm{Co}^{60}$ Gamma Irradiator at BREL. Above, sensors mounted in irradiator for testing

SEGR testing by bombardment of heavy ions was performed at Texas A\&M University's K500 Superconducting Cyclotron with ECR ion source in College Station, Texas. Sensors were mounted on a test fixture, shown in Figure 9, which allowed the hydrogen sensors to be electrically biased before and after the radiation dose. The sensor fixture and sensors were heated for the duration of the test. The sensors were subjected to ion beams of ${ }^{129} \mathrm{Xe}$ at two Linear Energy Transfer (LET) levels, 40.1 and $55.3 \mathrm{MeV}^{*} \mathrm{~cm}^{2} / \mathrm{mg}$, which is in the energy range of cosmic ray ions. The LET levels of the xenon ions are based on testing with silicon. The total fluence of ions for each sensor was at least 4.0 $\times 10^{6}$ particles $/ \mathrm{cm}^{2}$. In all cases, the sensors exhibited no performance shift during the tests. 




Figure 9: Sensors mounted for Heavy Ion Testing at Texas A\&M University K500 Superconducting Cyclotron.

Proton irradiation testing was performed at the 76" Isochronous Cyclotron at the Crocker Nuclear Laboratory on the campus of the University of California at Davis (Figure 10). Devices were tested with a $1 \%$ hydrogen/balance air calibration gas before and after exposure, but were not heated nor electrically biased during proton bombardment. Sensors were irradiated with a $61.6 \mathrm{MeV}$ proton beam (calculated value) with increasing fluence levels of $10^{9}$ and $10^{10}$ particles $/ \mathrm{cm}^{2}$. Five (5) sensors were tested at each fluence level. Up to fluence levels of $10^{\wedge 10}$ particles $/ \mathrm{cm} 2$, the sensors showed no shift in baseline or sensitivity.

\section{0-Day Calibration Test}

Since calibration data from the qualification program would not be available until late 2005 , it was decided to utilize a Flight Like Development Unit (FLDU) to gain preliminary data. The FLDU utilized a single die processed from flight hardware and as wells as the latest screening and calibration procedures. A baseline data set was established and the unit was operated continuously for a minimum of 90 days on a development OGA system at MSFC (Figure 11 and Figure 12) for the purposes of gaining

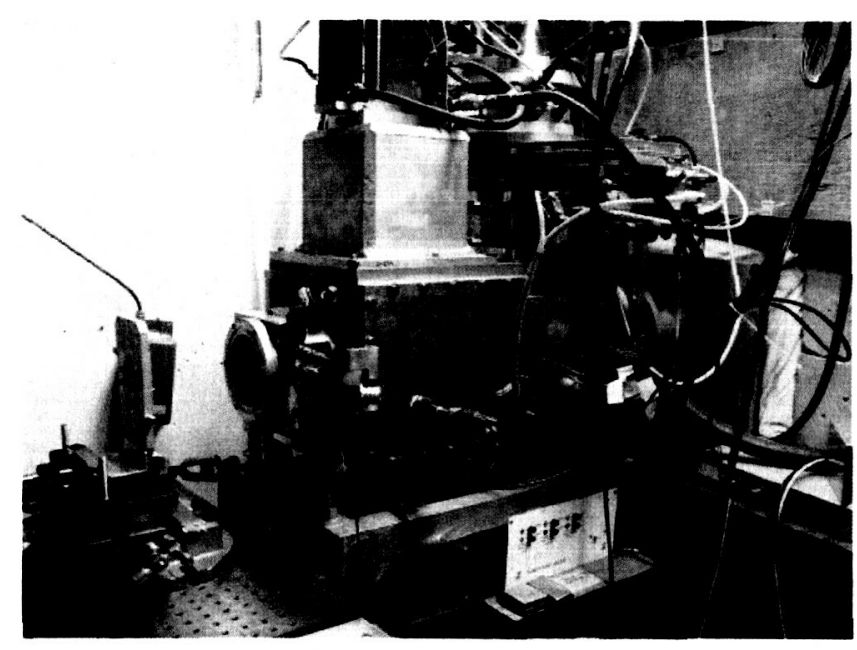

Figure 10: Proton irradiation testing at Crocker Nuclear Laboratory, UC Davis

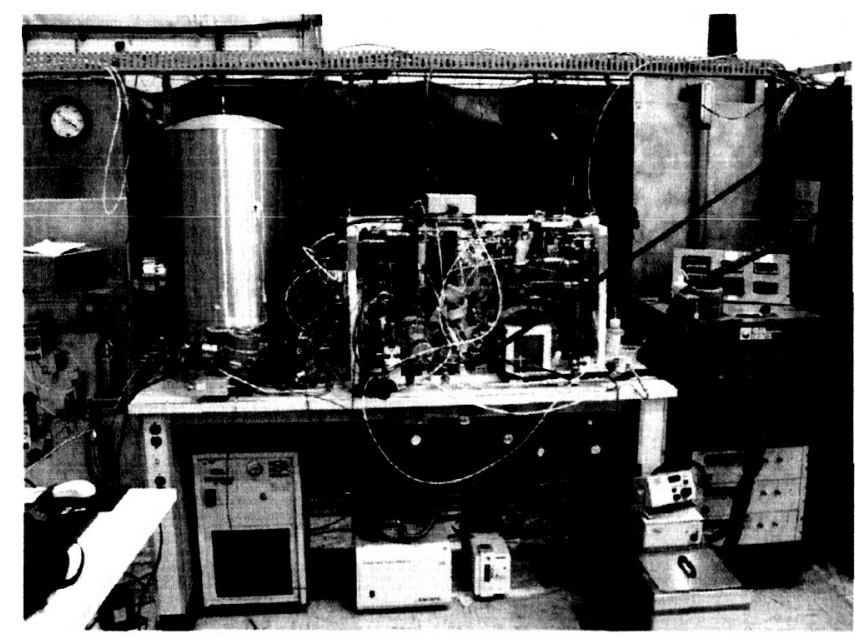

Figure 11: Development OGA System Test with FLDU at MSFC

operational time in a realistic system environment. 


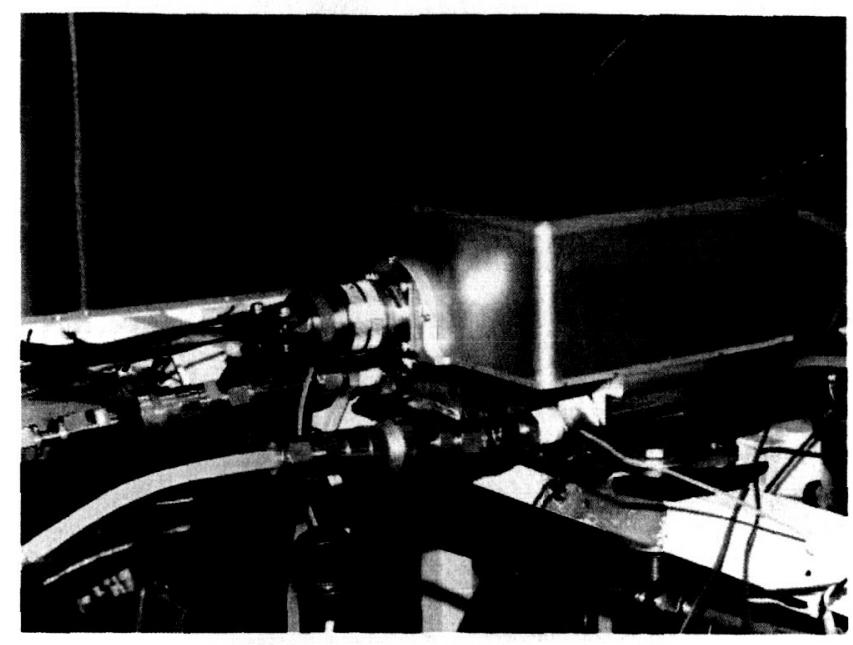

Figure 12: FLDU at MSFC

At the completion of 90 days, the hardware was returned to $\mathrm{MEI}$ to assess the performance in relation to the baseline data. As of this writing, the reassessment is in process at $\mathrm{MEI}$.

\section{Integration Tests at HSSSI}

The hydrogen sensor ORU was successfully integrated into the OGA system (Figure 13) at HSSSI. Hydrogen was injected into the oxygen flow path and the ORU demonstrated its effectiveness to detect a simulated leak in the system causing the system to take action. As part of the OGA system, the ORU has demonstrated compliance to ISS EMI conducted and susceptibility requirements and power quality testing as part of system level activity in June 2004.

\section{ON-ORBIT LOGISTICS}

\section{The 90-Day Calibration Cycle}

Presently the 90-day calibration cycle means that at the completion of 90 days of ground and or on-orbit cumulative operation, the Hydrogen Sensor ORU must be removed from the OGA system in order to perform a re-calibration. This interval of time was established in the early phase of development and was primarily driven by repeatability and humidity test results.

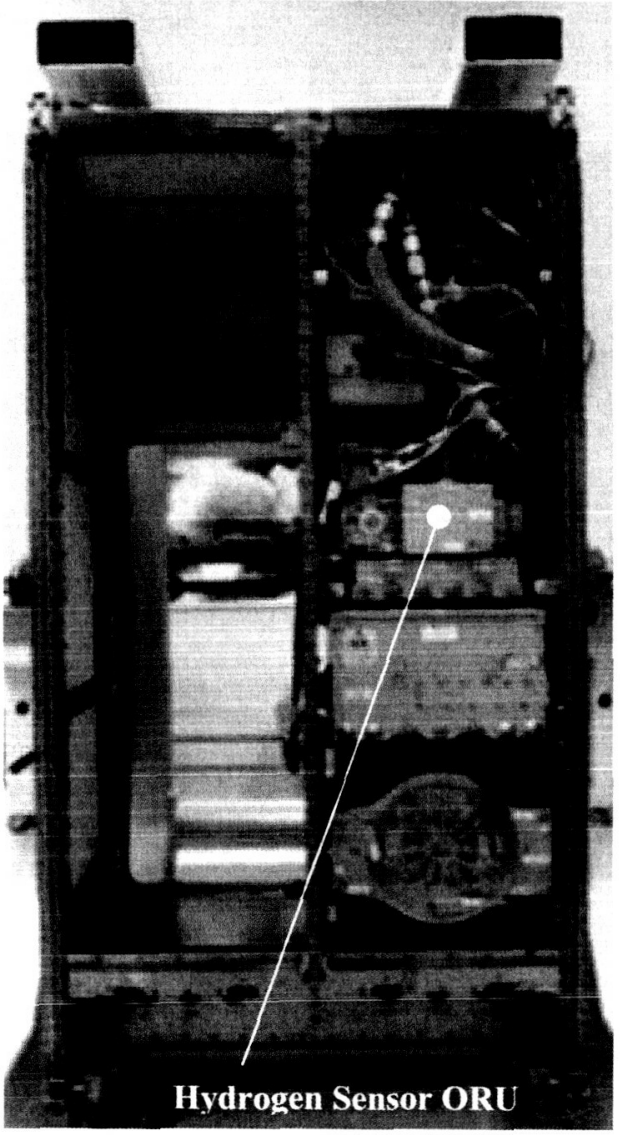

Figure 13: OGA System with Hydrogen Sensor Installed

In addition to FLDU testing, plans are being developed with MSFC and MEI to evaluate the feasibility of extending the 90-day calibration life to better facilitate the logistics of removing and replacing the ORU at every 90-day interval of operation.

The proposed plan would use the Qualification Unit to assess performance over multiple 90-day calibration cycles. With enough statistical data, there is reasonable belief that the calibration cycle can be lengthened. 


\section{Exposure to Moisture Work-Around}

As mentioned earlier, condensation of water in the hydrogen sensor ORU is not possible under normal operating conditions. While, it has been determined that there exists the possibility for condensed water to exist in the ORU under certain hardware shut down modes, this has necessitated ground and on-orbit work-arounds.

\section{Future Sensor Development Planned}

Currently work is underway on further improvements to the sensing element die for this application. Specifically modifications to the die structure are being evaluated to reduce the effect of humidity on the signal and to eliminate the potential for electrolysis on the surface of the die. These improvements are expected to be completed in 2005 and potentially be available to the program.

\section{CONCLUSION}

The Hydrogen Sensor ORU has progressed from the development stage to flight hardware and during that time substantial improvements in performance have been made. Understandings how the sensor performed under OGA environments was key those improvements. Sensitivity to moisture had represented a technical challenge to the MEI-HS team and this effect was significantly reduced through changes in the sensor's operation, calibration and screening procedures resulting in repeatable data over the expected humidity range.

Testing of the FLDU is intended to help assess the potential of extending the present 90-day calibration cycle. Additional tests are planed with the qualification unit to further evaluate extending this time through performance assessments over multiple 90-day calibration cycles. The overall goal is to reduce the frequency of on-orbit change outs of the Hydrogen Sensor ORU.

Development activity is underway to further reduce the sensitivity due to humidity and to eliminate the electrolysis effect when the sensing element is in contact with liquid water and may be available to the program. Two flight units have been delivered to HSSSI and two more are expected by August 2005. 


\section{REFERENCES}

1. Hunter, G.W., Liu, C.C., Makel, D., 2001, MEMs Handbook, CRC Press LLC, ed. M. Gadel-Hak, Ch. 22.

2. Hunter, G.W., Neudeck, P. G., Fralick, G., Makel, D., Liu, C.C., Ward, B., Wu, Q. H., Thomas, V., Hall, G., 2001, "Microfabricated Chemical Sensors For Space Health Monitoring Applications," AIAA 20014689.

3. Seeling, E., Makel, D.,"Hydrogen Sensor for the ISS Oxygen Generation Assembly", SAE 2002-01-2406

\section{CONTACT}

George Msadoques

One Hamilton Rd, M/S TBD

Windsor Locks, CT 06096-1010

Phone: 860-654-1386

Fax: (860) 660-1933

E-mail: george.msadoques@hs.utc.com

Darby Makel Makel Engineering, Inc Chico, CA 95973

Phone: 530-895-2771

Fax: 530-895-2777

E-mail: dmakel@makelengineering.com 